\title{
The nature of the manifestation of procrastination, level of anxiety and depression in medical students in a period of altered psycho-emotional state during forced social distancing because of pandemic COVID-19 and its impact on academic performance
}

\author{
Ivan Romash \\ Ivano-Frankivsk National Medical University, Ivano-Frankivsk, Ukraine
}

\begin{abstract}
Introduction: The fact that learning is moving online is probably a disappointment to most medical students. This is especially true for middle and senior students, in whom most disciplines are clinical and focus on practical work with patients. During the period of forced social distancing and online learning in connection with the COVID-19 pandemic, such a common way of learning became impossible. All these changes obviously affect the mental health of medical students, who are already at risk for such phenomenon as procrastination and disorders as anxiety and depression.
\end{abstract}

Purpose: Investigate and evaluate the level of anxiety, depression, and the nature of the manifestation of procrastination in medical students Faculty of Medicine and the Faculty of Training Foreign Citizens (FTFC) of Ivano-Frankivsk National Medical University (IFNMU) in a period of altered psycho-emotional state during the period of forced social distancing and its impact on academic performance.

Methodology: 212 medical students were interviewed. The survey was voluntary and anonymous. We used two clinical test methods to identify emotional disturbances: the Hospital Anxiety and Depression Scale (HADS) and the Montgomery-Asberg Depression Rating Scale (MADRS) (Zigmond A.S. et.al.,1983; Svanborg P., 2001). In order to assess the propensity of students to procrastinate, we also used the "Questionnaire to study the propensity of the individual to procrastination" (Shivari ,O.A., 2015), which is a modification of the "General Procrastination Scale" (Lay, C., 1986) and consists of two scales: "Personally conditioned procrastination scale" (Scale I) and "Situationally conditioned procrastination scale" (Scale II). The statistical analysis of the results was performed using STATISTICA 7.0 software packages and the package of statistical functions of Microsoft Excel.

Results and Discussion: During the testing, students note that the most important problem for them is time management. The analysis of the results survey by "Personally conditioned procrastination scale" (Scale I) "showed that 15\% of the students have a low, $47.5 \%$ - medium, $37.5 \%$ - high level of personal propensity to procrastination. The analysis of the results "Situationally conditioned procrastination scale" (Scale II) showed that $25 \%$ of students have a low level of situational procrastination, $37.5 \%$ - medium and $37.5 \%$ - high, respectively. The study of the relationship between the level of propensity to procrastination (Scale I) and academic performance in medical students showed that in the group with low procrastination the success rate is higher than in groups with medium and high procrastination $r=-0.58$; $p$ $<0.05$ ). We also established a direct correlation between the Scale II indicators and the 
Special Issue -

MHGC Proceeding 2020

average academic performance indicators. After the survey and analysis of the results on the HADS and MADRS scales, we found a clear relationship between increased learning during the period of altered psychoemotional state and the severity of anxiety-depressive symptoms in medical students, which manifested itself in an increase in the level of anxiety and mood disorders of varying degrees.

Conclusions: Thus, procrastination that occurs in the surveyed medical students in the period of altered psycho-emotional state during forced social distancing because of pandemic COVID19 has had "situational" situational nature. Besides, the study confirmed that the pandemic of COVID-19, as a stressful moment of the external test of identity, caused the emergence of protective mechanisms in medical students in the form of emotional and behavioral changes and disorders. The results obtained indicate the presence of adjustment disorders in the period of the altered psychoemotional state during forced social distancing because of pandemic COVID-19 in all the studied groups. Medical students also need to be made aware that depression is not a cause for shame. Future doctors should be able to maintain their mental and emotional health, as well as know, how to deal with classmates who suffer from mental illness.

\section{Keywords}

forced social distancing, pandemic COVID-19, procrastination, anxiety, depression, medical students.

Address for correspondence:

Ivan Romash, Ivano-Frankivsk National Medical University, Ivano-Frankivsk, Ukraine. e-mail: ivromash@ifnmu.edu.ua

This work is licensed under a Creative Commons Attribution-

NonCommercial 4.0 International License (CC BY-NC 4.0).

\section{(c) (i) (9)}

(c) Copyright: Romash, 2020

Submitted for publication: 23 May 2020

Received: 23 May 2020

Accepted for publication: 15 October 2020

Licensee NDSAN (MFC- Coordinator of the NDSAN), Italy DOI: http://doi.org/10.32437/mhgci.v4i2.92

\section{Introduction}

Studies by Beutel et al have shown that the most prone to procrastination are the unemployed, single, and students, although it is common to all age groups. Every day, medical students face problems such as delaying module preparation, writing medical histories on the last night, sleepless days and nights before passing important exams - all of which are manifestations of procrastination. Procrastination (from the Latin "procrastinare") - a psychological term that means a person's tendency to postpone unpleasant tasks for later, the attraction to things that bring more pleasure or faster results. And in 2020, they also faced forced social distancing in connection with COVID-19.

According to scientific literature, depression is a disease with a high prevalence and a tendency to chronicity. Depression - a pandemic of the 21-st century - is one of the world health organization's (WHO's) main focuses. According to the Global Burden of Disease Study (GBD) 2017, the incidence of disability among young people due to the mild, moderate, or severe depressive episode was $0.145,0.396,0.658$, respectively (IHME, 2018). According to WHO statistics, in highdeveloping countries, the suicide rate is the highest, accounting for 11.5 cases per 100,000 population and continues to rise, ranking second after accidents, among the causes of deaths in people aged 15 to 29 years (WHO, 2014). Therefore, in the 2013-2020 Comprehensive Mental Health Action Plan adopted by the 66-th World Health Assembly, WHO Member States have committed themselves to work towards a global goal of reducing the suicide rate in countries by $10 \%$ by 2020 .

Scientists have shown that medical training itself is a risk factor for the onset and maintenance of symptoms of depression. Also, some scientific papers have reported that various processes associated with competition in medical schools, the first and subsequent contacts with death and pathological processes, fear of acquiring diseases caused emotional 
Special Issue -

MHGC Proceeding 2020

experiences in medical students. According to other scientific data, medical students are dreamy, idealistic in the study, inadequately assess their capabilities. As a result, the dissatisfaction of learning is formed, and their indifference to their responsibilities is growing. There are signs of depersonalization that indicate the development of emotional burnout, depression. According to scientific data, medical students are more likely to experience depression than others. The saddest part is that they find this condition uncharacteristic for the doctors and therefore underestimate it. This conclusion was reached by Lisa $S$. Rotenstein et al (2016) in a meta-analysis of nearly 200 studies involving 129,000 medical students from 47 countries. According to the authors of this study, the prevalence of depression or depressive symptoms among medical students was $27.2 \%$, suicidal tendencies - 11.1\%. However, only a small proportion of students who were screened for depression were asked for help. Sergio Baldassini, who heads a group of scientists who conducted a study of 481 medical students at a private medical school in Brazil, also noted, that they often suffered from depression, especially during internships. In his research, he revealed the scope of the problem and made a detailed analysis of the symptoms. Another important pattern has been found in the research of Karina Pereira-Lima and co-authors, physicians with positive screening for depressive symptoms are at greater risk of medical error.

\section{Purpose}

To investigate and evaluate the level of anxiety, depression, and the nature of the manifestation of procrastination in medical students in a period of altered psycho-emotional state during the period of forced social distancing and its impact on academic performance.

\section{Methodology}

To achieve the goal of the study, 212 medical students Faculty of Medicine and the Faculty of Training Foreign Citizens (FTFC) of Ivano-Frankivsk National Medical University (IFNMU)aged 18 to 25 were interviewed, among them 52 were boys and 160 girls. The survey of students was carried out during the preparation of students for tests, exams, final modular controls, that is, at the period of an altered psycho-emotional state during the pandemic of COVID-19. We studied the effect of intensification of learning on the level of anxiety and stress in students.

The research was approved by the Bioethics Committee of Ivano-Frankivsk National Medical University and was conducted according to the principles of the Helsinki Declaration. All patients signed a voluntary informed consent before the study.

The survey was voluntary and anonymous. We used two clinical test methods to identify emotional disturbances: the Hospital Anxiety and Depression Scale (HADS) and the MontgomeryAsberg Depression Rating Scale (MADRS) (Zigmond A.S. et.al., 1983; Svanborg P., 2001). In order to assess the propensity of students to procrastinate, we also used the "Questionnaire to study the propensity of the individual to procrastination" (Shivari ,O.A., 2015), which is a modification of the "General Procrastination Scale" (Lay, C., 1986) and consists of two scales: "Personally conditioned procrastination scale" (Scale I) and "Situationally conditioned procrastination scale" (Scale II). Scale I indicates the general level of procrastination, and Scale II motivational procrastination, its high indicators suggest that the questionnaire will perform some work only in the presence of motivation. Separately, the suicidal tendency was assessed based on the analysis of data on the tenth question in the MADRS scale.

The statistical analysis of the results was performed using STATISTICA 7.0 software packages and the package of statistical functions of Microsoft Excel. Arithmetic mean (M) and standard error $( \pm m)$ were used to describe the quantitative characteristics. The reliability of the obtained indices was confirmed by calculating the errors for relative values, and the probability of the difference in the data in the comparable groups was proved on the basis of the calculation of t-coefficient (Student t-test) and determination of precise prognosis according to the accuracy table.

\section{Results and Discussion}

During the testing, students note that the most important problem for them is time management. Unlike traditional classes at university departments, where the meeting is held in certain rooms, on a regular schedule and they know exactly where and when they have classes, and they can somehow organize their schedule around it, most online learning is perceived differently for them. But they still need to manage their time and participate in coursework and 
Special Issue -

MHGC Proceeding 2020

understand that they have a responsibility to determine the time when they will do their academic work.

The analysis of the results survey by "Personally conditioned procrastination scale" (Scale I) "showed that $15 \%$ of the students have a low, $47.5 \%$ - medium, $37.5 \%$ - high level of personal propensity to procrastination (Fig. 1).

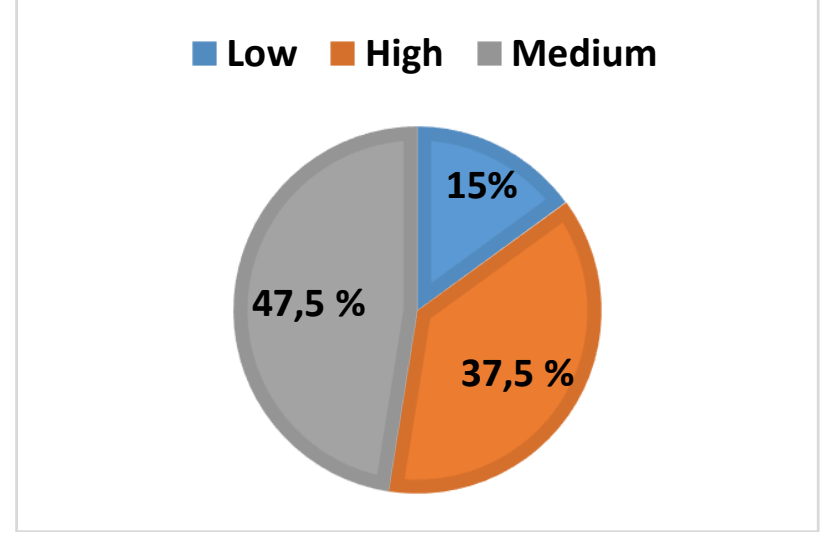

Fig. 1 Distribution of students depending on the level of manifestation general procrastination.

The analysis of the results "Situationally conditioned procrastination scale" (Scale II) showed that $25 \%$ of students have a low level of situational procrastination, $37.5 \%$ - medium and $37.5 \%$ - high, respectively (Fig. 2)

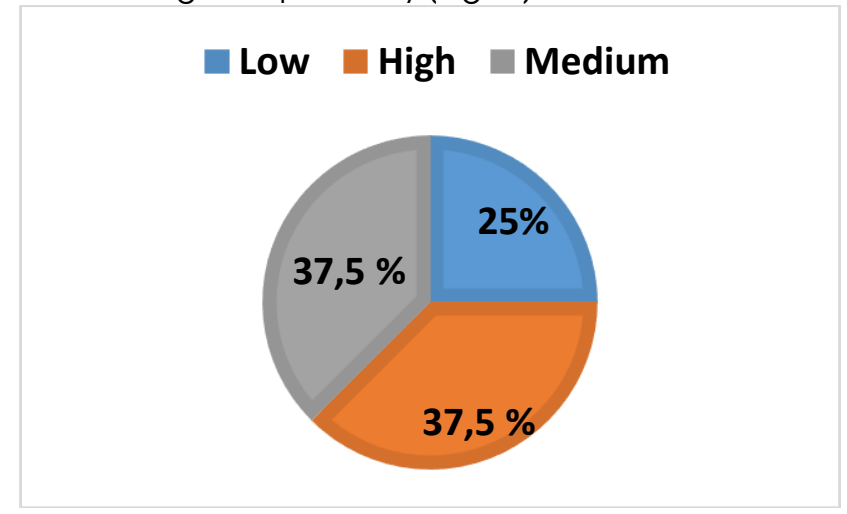

Fig. 2 Distribution of students depending on the level of manifestation situational procrastination.

The study of the relationship between the level of propensity to procrastination (Scale I) and academic performance in medical students showed that in the group with low procrastination the success rate was higher than in groups with medium and high procrastination ( $r=-0.58 ; \mathrm{p}$ $<0.05$ ). We also established a direct correlation between the Scale II indicators and the average academic performance indicators.

After the survey and analysis of the results on the HADS and MADRS scales, we found a clear relationship between increased learning during the period of altered psychoemotional state and the severity of anxiety-depressive symptoms in medical students, which manifested itself in an increase in the level of anxiety and mood disorders of varying degrees

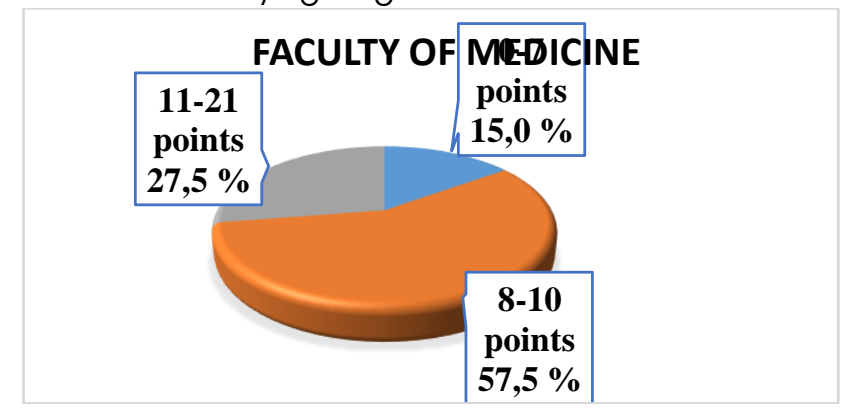

\section{FACULTY OF TRAINING FOREIGN CITIZENS}

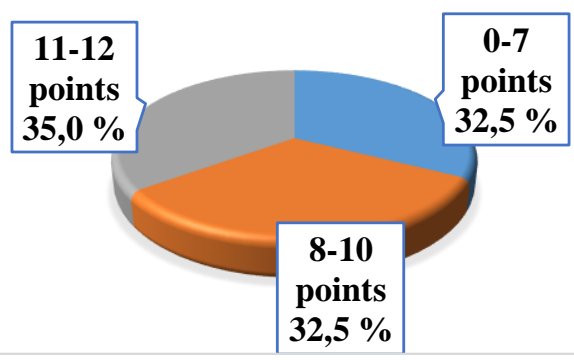

Fig. 3 Distribution of students depending on the level of manifestation of anxiety-depressive symptoms (according to the HADS scale).

Based on the results of the survey on the HADS scale during the period of altered psychoemotional state, a normal level of anxietydepressive symptoms (0-7 points on a scale) was found in $15 \%$ of students of Faculty of Medicine and $32.5 \%$ in students of FTFC. Symptoms of "borderline" states (8-10 points on a scale) were found in $57.5 \%$ and $32.5 \%$ of students of Faculty of Medicine and FPIG, respectively. Clinically pronounced anxiety and depression (11-21 points) were found in $27.5 \%$ of medical students and $35.0 \%$ of foreign students (Fig. 3)

In the gender distribution, among students of the Faculty of Medicine, the normal level of anxiety and depression during preparation for the delivery of modular controls is more common among females, accounting for 27.5\%, compared with $17.5 \%$ among males. It should be noted that the indicators of the symptoms of 
Special Issue -

MHGC Proceeding 2020

"borderline" states and the level of clinically expressed anxiety and depression were higher in male students and amounted to $65.0 \%$ and $25.8 \%$, respectively. In female students "borderline" states are noted in $44.1 \%$ of cases, and clinically expressed anxiety-depressive symptoms in $27.5 \%$.

As for the students of FTFC, the picture of gender distribution is somewhat different, namely: the symptoms of "borderline" states were higher in girls - 44.0\%, while in men - 27.5\%. However, men representatives among foreign students had a high level of clinically expressed anxiety and depression - 38.45\%, while among women students it was lower - 32.5\%
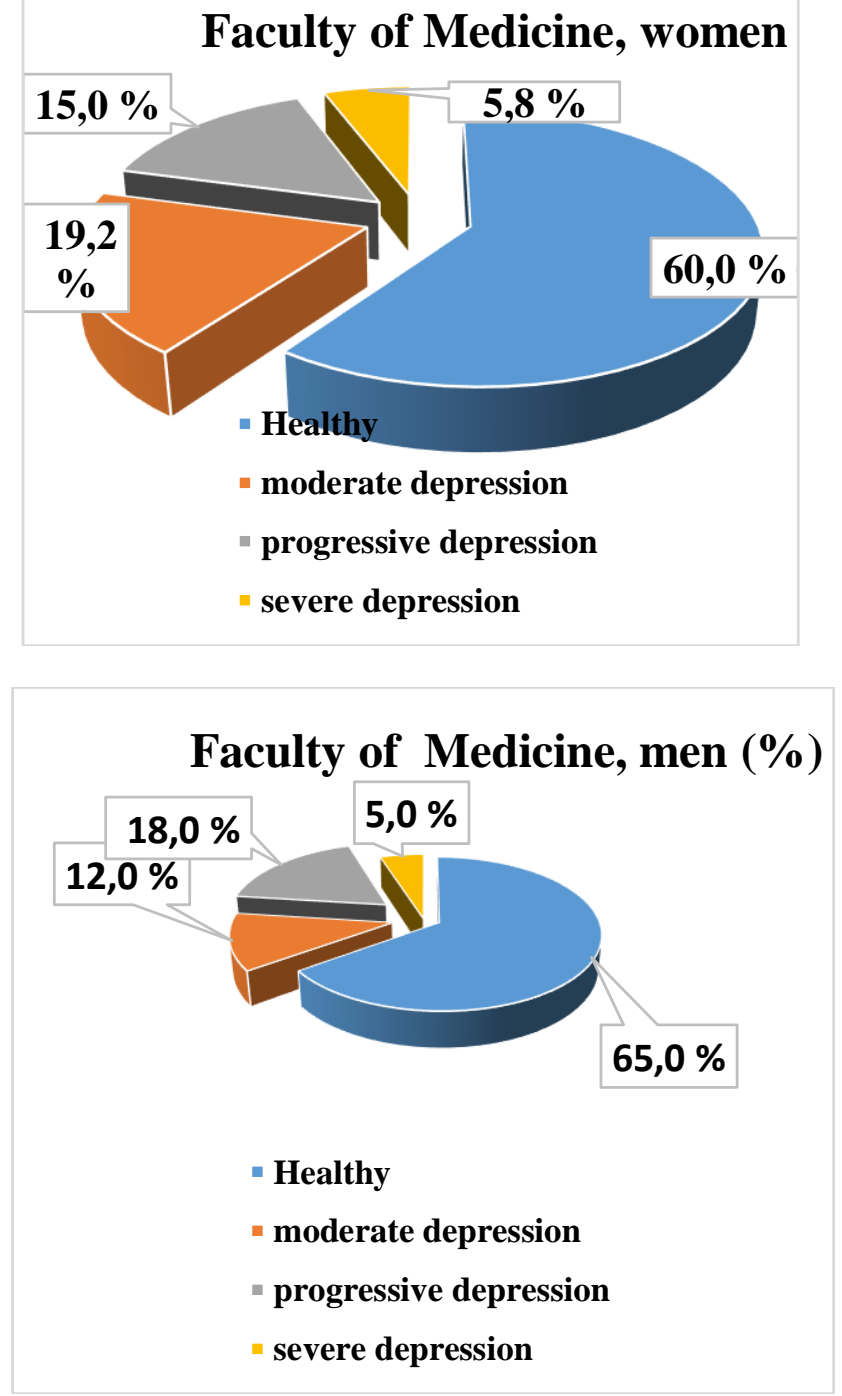

Fig 4. Distribution of the level of manifestation of depression in students of the Faculty of medicine by gender (according to the MADRS scale data).

According to a survey using the clinical test method MADRS (Fig.4), indicators of the norm, that is, the absence of depressive symptoms, were found in $60.0 \%$ of women and in $65.0 \%$ of men of the Faculty of Medicine. Signs of moderate depression were found in 19.2\% of women and $12.0 \%$ of men. Progressive depression in both sexes manifested itself, almost at the same level: $15.0 \%$ in women and $18.0 \%$ in men. It should be noted that the symptoms of severe depression or major depressive episode were observed in men and amounted to $5.0 \%$ of cases, while among women $-5.8 \%$
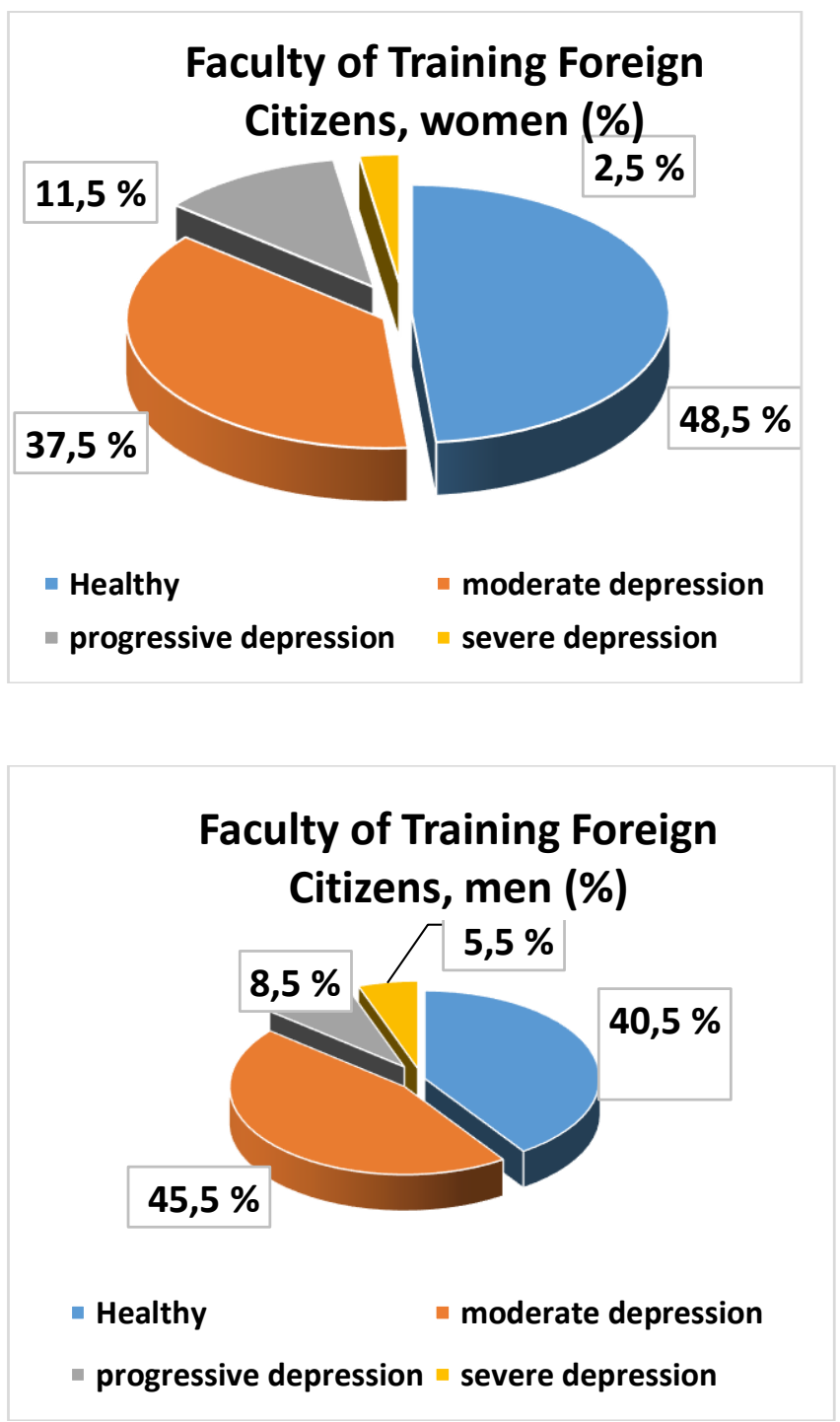

Figure 3. Distribution of the level of depression in students of Faculty of Training Foreign Citizens by gender (according to the MADRS scale).

After processing the data of the survey of foreign students, we received the following indicators: there were no depressive symptoms in $48.5 \%$ of female students and in $40.5 \%$ of male 
Special Issue -

MHGC Proceeding 2020

students; we found signs of moderate depression in $37.5 \%$ of women and $45.5 \%$ of men; progressive depression - $11.5 \%$ among females and $8.5 \%$ among males. The symptomatology of severe depression or a major depressive episode was observed among $2.5 \%$ and $5.5 \%$ respectively.

According to our study, medical students, thought that they should not tell others about their mental state. This study found that medical students are not seeking help because they are afraid of becoming less valuable to society. The results obtained by us coincide with the data Sergio Baldassini et.al. (2012) and Lisa S. Rotenstein et al. (2016)

\section{Conclusions}

Thus, procrastination that occurs in the surveyed medical students in the period of altered psycho-emotional state during forced social distancing because of pandemic COVID19 has had a "situational" nature.

Besides, the study confirmed that the pandemic of COVID-19, as a stressful moment of the external test of identity, caused the emergence of protective mechanisms in medical students in the form of emotional and behavioral changes and disorders. The results obtained indicate the presence of adjustment disorders in the period of the altered psychoemotional state during forced social distancing because of pandemic COVID-19 in all the studied groups.

Medical students also need to be made aware that depression is not a cause for shame. Future doctors should be able to maintain their mental and emotional health, as well as know, how to deal with classmates who suffer from mental illness.

\section{Conflict of interest}

Author declares that he has no conflict of interests

\section{References}

Baldassin S. (2012). Depression in medical students: Cluster symptoms and management [text] / Baldassin S., Silva N., Correa de Toledo T. Ferraz Alves et.al. // Journal of Affective Disorders. 150(1).

Baldassin, S., Silva, N., de Toledo Ferraz Alves, T. C., Castaldelli-Maia, J. M., Bhugra, D., Nogueira-Martins, M. C., de Andrade, A. G., \&
Nogueira-Martins, L. A. (2013). Depression in medical students: cluster symptoms and management. Journal of affective disorders, 150(1), $110-114$. https://doi.org/10.1016/j.jad.2012.11.050

Fleischmann, A., Arensman, E., Berman, A., Carli, V., De Leo, D., Hadlaczky, G., Saxena, S. (2016). Overview evidence on interventions for population suicide with an eye to identifying best-supported strategies for LMICs. Global Mental Health, 3, E5. doi: 10.1017/gmh.2015.27

Institute for Health Metrics and Evaluation (IHME) (2018). Findings from the Global Burden of Disease Study 2017 [text] / Seattle, WA: IHME. $25 \mathrm{~S}$.

Pereira-Lima, K., Mata, D. A., Loureiro, S. R., Crippa, J. A., Bolsoni, L. M., \& Sen, S. (2019). Association Between Physician Depressive Symptoms and Medical Errors: A Systematic Review and Meta-analysis. JAMA network open, 2(11), el916097. https://doi.org/10.1001/jamanetworkopen. 201 9.16097

Pereira-Lima, K., Mata, D. A., Loureiro, S. R., Crippa, J. A., Bolsoni, L. M., \& Sen, S. (2019). Association Between Physician Depressive Symptoms and Medical Errors: A Systematic Review and Meta-analysis. JAMA network open, 2(11), el916097. https://doi.org/10.1001/jamanetworkopen. 201 9.16097

Preventing suicide: a global imperative. [text] (2014) / Geneva: World Health Organization.97 S.

Rotenstein, L. S., Ramos, M. A., Torre, M., Segal, J. B., Peluso, M. J., Guille, C., Sen, S., \& Mata, D. A. (2016). Prevalence of Depression, Depressive Symptoms, and Suicidal Ideation Among Medical Students: A Systematic Review and Meta-Analysis. JAMA, 316(21), 2214-2236. https://doi.org/10.1001/jama.2016.17324

Tichenor, M., \& Sridhar, D. (2019). Metric partnerships: global burden of disease estimates within the World Bank, the World Health Organisation and the Institute for Health Metrics and Evaluation. Wellcome open research, 35.https://doi.org/10.12688/wellcomeopenres. 15011.2

Zigmond A.S., Snaith RP. The hospital anxiety and depression scale [text] /A.S. Zigmond, R.P Snaith //Acta Psychiatr Scand.- 1983.- 67(6).S.361-70 